\title{
CARACTERIZAÇÃO DOS SÓLIDOS INTERSTICIAIS DE SISTEMAS ALAGADOS CONSTRUÍDOS DE ESCOAMENTO HORIZONTAL SUBSUPERFICIAL
}

Gheila Corrêa Ferres Baptestini ${ }^{1}$, Antonio Teixeira de Matos $^{2}$, Paulo Roberto Cecon ${ }^{3}$, Suymara Toledo Miranda ${ }^{4}$

\section{RESUMO}

Com a realização deste estudo, objetivou-se avaliar o efeito da inversão no sentido do escoamento em sistemas alagados construídos de escoamento horizontal subsuperficial (SACs-EHSS) nas características qualitativas e quantitativas do material colmatante do meio poroso. Para isso, ao fim do período experimental, foram coletadas amostras do leito de seis SACs-EHSS: dois não cultivados (SACs-EHSS 1 e 4), dois cultivados com capim tifton 85 (Cynodon spp.) (SACs-EHSS 2 e 5) e dois cultivados com alternantera (Alternanthera philoxeroides) (SACs-EHSS 3 e 6). Das amostras retiradas do material poroso dos SACs foram separados e analisados os sólidos que ocupavam os poros. Nesse material foram quantificadas a massa de sólidos totais $\left(\mathrm{C}_{\mathrm{ST}}\right)$, sólidos voláteis totais $\left(\mathrm{C}_{\mathrm{SVT}}\right)$ e sólidos fixos totais $\left(\mathrm{C}_{\mathrm{SFT}}\right)$ por volume de meio poroso $\left(\mathrm{kg} \mathrm{m}^{-3}\right)$ dos SACs-EHSS. Cada espécie vegetal influenciou de modo distinto a distribuição dos $\mathrm{C}_{\mathrm{ST}}, \mathrm{C}_{\mathrm{SFT}}$ e $\mathrm{C}_{\mathrm{SVT}}$ nos SACs-EHSS cultivados. Mesmo em condições de relação $\mathrm{C}_{\mathrm{SVT}} / \mathrm{C}_{\mathrm{ST}}$ baixa a fração orgânica dos sólidos proporcionou estruturação do material suporte superficial dos SACs-EHSS. A inversão no sentido do escoamento não proporcionou redução no acúmulo de matéria orgânica no meio poroso. O alto grau de colmatação dos SACs-EHSS impediu ou tornou menos perceptíveis e prolongados os efeitos positivos da inversão no sentido do escoamento na condição do meio poroso.

Palavras-chave: colmatação, entupimento de poros, tratamento de águas residuárias

\section{ABSTRACT \\ CHARACTERIZATION OF THE INTERSTITIAL SOLIDS IN CONSTRUCTED WETLANDS OF SUBSURFACE HORIZONTAL FLOW}

The objective of the present study was to evaluate the effect of reversing the direction of flow in constructed wetland of subsurface horizontal flow (CW-HSSF) in the qualitative and quantitative characteristics of the clogging material. For this, in the end of the experimental period, bed samples were collected from six CW-HSSF: two non-cultivated (CW-HSSF 1 and 4), two cultivated with tifton 85 grass (Cynodon spp.) (CW-HSSF 2 and 5) and two cultivated with alternathera (Alternanthera philoxeroides) (CW-HSSF 3 and 6). The interstitial solids were separated from the samples taken from the CW-HSSF porous media. In this material was quantified the mass of total solids $\left(\mathrm{C}_{\mathrm{ST}}\right)$, total volatile solids $\left(\mathrm{C}_{\mathrm{SVT}}\right)$, and fixed total solids $\left(\mathrm{C}_{\mathrm{SFT}}\right)$ per volume $\left(\mathrm{kg} \mathrm{m}^{-3}\right)$ of $\mathrm{CW}-\mathrm{HSSF}$ porous media. Each plant species influenced differently the distribution of $\mathrm{C}_{\mathrm{ST}}, \mathrm{C}_{\mathrm{SFT}}$ and $\mathrm{C}_{\mathrm{SVT}}$ in planted $\mathrm{CW}-\mathrm{HSSF}$. Even in conditions of $\mathrm{C}_{\mathrm{SVT}} / \mathrm{C}_{\mathrm{ST}}$ low relationship the organic fraction provided structure of the surface material support of CW-HSSF. On average, the reversal in the flow direction does not reduced the accumulation of organic matter in porous media. The high degree of clogging in CW-HSSF prevented or made less noticeable and prolonged the positive effects of reversing the direction of flow of wastewater on conditions of the porous media.

Keywords: clogging, blocking of pores, wastewater treatment

Recebido para publicação em 31/05/2015. Aprovado em 01/12/2015.

1 - Engenheira Agrícola, gheilacf@yahoo.com.br

2 - Engenheiro Agrícola, Professor Titular da UFMG/Belo Horizonte-MG, atmatos@ufv.br

3 - Agrônomo, Professor Titular da UFV/Viçosa-MG, cecon@ufv.br

4 - Engenheira Agrícola, suymara-miranda@hotmail.com

562 REVENG $562-574 p$.

ENGENHARIA NA AGRICULTURA, VIÇOSA - MG, V.23 N.6, NOVEMBRO / DEZEMBRO 2015 


\section{INTRODUÇÃO}

Os sistemas alagados construídos de escoamento horizontal subsuperficial (SACs-EHSS), utilizados no tratamento de águas residuárias, são sistemas bastante dinâmicos, nos quais ocorrem muitas transformações ao longo do tempo. Como resultado dos processos físicos, químicos e biológicos durante o tratamento da água residuária, há acúmulo de sólidos no meio poroso resultando em gradual colmatação, denominação que se dá ao processo de entupimento do meio poroso (NIVALA et al., 2012), um dos principais problemas associados aos SACs-EHSS.

A colmatação é mais acentuada na parte inicial do sistema, que é a que tem o primeiro contato com a água residuária afluente, agindo como um filtro e retendo grande parte dos sólidos suspensos presentes na água residuária (KNOWLES et al., 2011). Com o sistema em operação, o entupimento dos poros tem avanço progressivo em direção à sua saída, formando um gradiente longitudinal de ocupação do meio poroso. Em SACs-EHSS, pode ocorrer também um gradiente vertical de distribuição dos sólidos ocupantes do meio poroso, com maior acúmulo na camada superficial. Este perfil parece ser motivado pelo crescimento das raízes das plantas e deposição de sólidos sobre o substrato, oriundos da água aplicada e dos restos dos vegetais cultivados nesses sistemas (CHAZARENC; MERLIN, 2005; PEDESCOLL et al., 2009; KNOWLES et al., 2011).

De acordo com PEDESCOLL et al. (2009), a quantidade e a composição dos sólidos que se acumulam nos interstícios do meio poroso, também denominado de material colmatante, varia de acordo com cada sistema, devido aos diferentes formatos, formas de manejo, características da água residuária e condições ambientais às quais estão submetidos. Além disso, a distribuição da colmatação e a velocidade com que ela ocorre no SAC-EHSS estão relacionadas com a concepção e operação do sistema (KNOWLES et al., 2011).

Embora o conceito de colmatação seja simples, o processo envolve muitos fatores que ainda não estão totalmente compreendidos (KADLEC; WALLACE, 2008; KNOWLES et al., 2011). Dessa forma, para que se possam propor técnicas operacionais adequadas, existe a necessidade de maior entendimento acerca da dinâmica do processo de entupimento dos poros e da forma que esse entupimento pode ser retardado ou, até mesmo, retrocedido.

A proposição de invertero sentido do escoamento da água residuária, como ação descolmatante, baseia-se na hipótese de que a parte inicial do SAC-EHSS, com alto índice de entupimento de poros, passaria a receber uma água residuária que já passou por um processo inicial de depuração e que apresenta grande concentração de nutrientes disponíveis. Isso estimularia os microrganismos a degradarem o material colmatante e as plantas a mineralizar o material orgânico e remover o inorgânico. Assim, os microrganismos e as plantas atuariam como agentes descolmatantes do sistema.

Uma das maneiras de avaliar alterações e grau de colmatação do meio poroso, decorrentes da inversão no sentido do escoamento da água residuária, é a de caracterização dos sólidos intersticiais. Segundo NIVALA et al. (2012), este tipo de análise pode elucidar o grau e a natureza da obstrução/desobstrução.

Diante do exposto, teve-se por objetivo, com a condução deste trabalho, avaliar o efeito da inversão no sentido do escoamento em sistemas alagados construídos de escoamento horizontal subsuperficial (SACs-EHSS) nas características qualitativas e quantitativas dos sólidos retidos no meio poroso.

\section{MATERIAL E MÉTODOS}

O experimento foi conduzido na Universidade Federal de Viçosa (UFV), Viçosa/MG. A estrutura experimental foi constituída por seis SACs-EHSS, mantidos em casa de vegetação, utilizados no tratamento de água residuária de suinocultura (ARS). Estes sistemas estavam em operação há aproximadamente 2 anos e 3 meses quando foi feita a coleta de dados do presente estudo.

Os SACs-EHSS foram constituídos por caixas de fibra de vidro, em escala piloto, nas dimensões de $0,6 \mathrm{~m} \times 0,5 \mathrm{~m} \times 2,0 \mathrm{~m}$ de altura, largura e comprimento, respectivamente, assentados sobre o solo, sem declividade de fundo. Como substrato foi utilizada brita gnáissica $\left(\mathrm{D}_{60}=9,1 \mathrm{~mm}\right.$, coeficiente 
de uniformidade $-\mathrm{CU} \mathrm{D}_{60} / \mathrm{D}_{10}=3,1$ e volume de vazios inicial de $0,398 \mathrm{~m}^{3} \mathrm{~m}^{-3}$ ), com a qual foram preenchidos os SACs-EHSS até a altura de $0,55 \mathrm{~m}$, sendo o nível d'água mantido a $0,05 \mathrm{~m}$ abaixo da superfície do substrato.

$\mathrm{Na}$ saída de cada unidade experimental foi instalado um sistema de drenagem composto por tubos de PVC de $32 \mathrm{~mm}$ de diâmetro. Para possibilitar a inversão no sentido do escoamento, em três das unidades experimentais foram instaladas uma segunda entrada e uma segunda saída (SACs-EHSS 1, 2 e 3).

As espécies vegetais cultivadas foram 0 capim tifton 85 (Cynodon spp.) (SAC-EHSS $2 \mathrm{e}$ SAC-EHSS 5) e a alternantera (Alternanthera philoxeroides) (SAC-EHSS 3 e SAC-EHSS 6). Nos SAC-EHSS 1 e 4 não houve cultivo.

$\mathrm{Na}$ entrada de cada SAC-EHSS foi instalado um reservatório de $20 \mathrm{~L}$, equipado com uma torneira de PVC em seu fundo, que alimentava o sistema com a ARS. Diariamente, cada reservatório de 20 L era preenchido até sua máxima capacidade e só depois a ARS era aplicada nos SACs-EHSS. A ARS era homogeneizada antes de cada aplicação, a fim de garantir que os sólidos presentes na mesma fossem aplicados de maneira uniforme nos sistemas. A aplicação era intermitente, superficial e durava, em média, 2,0 h.

As faixas de valores das principais variáveis analisadas na ARS afluente aos SACs-EHSS, ao longo de todo período de tempo de condução do experimento, estão apresentadas no Quadro 1. As características operacionais dos SACs-EHSS, estabelecidas com base nas características da ARS, estão apresentadas no Quadro 2.

O critério para inversão no sentido do escoamento da água residuária baseou-se no monitoramento do avanço do escoamento superficial $(E S)$, efetuado medindo-se duas vezes por semana o alcance do mesmo, com auxílio de fitas métricas instaladas nas bordas dos sistemas. A inversão no sentido do escoamento foi efetuada quando se observou que o $E S$ da água residuária aplicada alcançou $50 \%$ do comprimento do SACEHSS, ou seja, 1,0 m, permanecendo assim, por no mínimo, quatro medidas sucessivas. Uma vez que isso não ocorreu no mesmo tempo, o sentido do escoamento da água residuária nos sistemas foi invertido em épocas diferentes.

Durante o período experimental, fez-se uma única inversão no sentido do escoamento em cada sistema, pois, desde o início do período de monitoramento do $E S$, o mesmo alcançou uma única vez o valor de $50 \%$ com comprimento do sistema. O SAC-EHSS 2, cultivado com o capim tifton 85 , foi o primeiro a ter seu escoamento invertido, o que ocorreu em jul/2012; o SAC-EHSS 1 , não cultivado, foi o segundo, o que ocorreu em nov/2012 e, por último, o SAC-EHSS 3, cultivado com alternantera, em set/2013.

Quadro 1. Valores médios e desvio padrão das principais variáveis da ARS afluente aos SACs-EHSS.

\begin{tabular}{cccccccccccc}
\hline pH & $\begin{array}{c}\mathrm{CE} \\
\left(\mathrm{dS} \mathrm{m}{ }^{-1}\right)\end{array}$ & DQO & DBO & ST & SVT & SST & NT & P & K & Na \\
\hline 7,46 & 5,741 & 5.529 & 2.220 & 4.363 & 2.419 & 2.111 & 675 & 127 & 350 & 84 \\
$\pm 0,30$ & $\pm 2,768$ & \pm 4.392 & \pm 1.548 & \pm 2.684 & \pm 1.504 & \pm 2.032 & \pm 411 & \pm 97 & \pm 258 & \pm 56 \\
\hline
\end{tabular}

Quadro 2. Características operacionais dos SACs-EHSS

\begin{tabular}{lllcc}
\hline $\begin{array}{c}\mathrm{Q} \\
\left(\mathrm{m}^{3} \mathrm{~d}^{-1}\right)\end{array}$ & $\begin{array}{c}\mathrm{Q}^{*} \\
\left(\mathrm{~m}^{3} \mathrm{~h}^{-1}\right)\end{array}$ & $\begin{array}{c}\mathrm{TDH} \\
(\mathrm{h})\end{array}$ & $\begin{array}{c}\mathrm{TCO}_{\mathrm{S}} \\
\left(\mathrm{g} \mathrm{m}^{-2} \mathrm{~d}^{-1} \mathrm{de} \mathrm{DBO}\right)\end{array}$ & $\begin{array}{c}\mathrm{TCO}_{\mathrm{T}} \\
\left(\mathrm{g} \mathrm{m}^{-2} \mathrm{~d}^{-1} \mathrm{de} \mathrm{DBO}\right)\end{array}$ \\
\hline 0,020 & 0,010 & 19,9 & $44,4 \pm 31,0$ & $177,6 \pm 123,8$ \\
\hline
\end{tabular}


Após a inversão no sentido do escoamento, a distribuição dos tratamentos foi feita da seguinte forma: SACs-EHSS controle, que não tiveram o sentido do escoamento invertido - SAC-EHSS 4ni, SAC-EHSS 5ni e SAC-EHSS 6ni; SACs-EHSS que tiveram o sentido do escoamento invertido SAC-EHSS 1i, SAC-EHSS $2 \mathrm{i}$ e SAC-EHSS $3 \mathrm{i}$.

Ao fim do período experimental, em out/2013, foram retiradas amostras do meio poroso do leito dos SACs-EHSS, com o objetivo de se quantificar e caracterizar os sólidos intersticiais. Para amostragem do meio poroso procedeu-se da seguinte forma:

1) Inicialmente, toda a água presente no interior dos SACs-EHSS foi retirada;

2) Após um período de $24 \mathrm{~h}$, foram coletadas três amostras ao longo do comprimento dos SACsEHSS, às distâncias de 0,02, 1,0 e 1,98 $\mathrm{m}$ do início do sistema (pontos 1, 2 e 3 , respectivamente, tendo como referência os sistemas não invertidos). Cada amostra compreendia todo material ao longo do perfil. Para os sistemas cultivados, o material coletado de 0 a $20 \mathrm{~cm}$ de profundidade foi separado do material coletado de 20 a $55 \mathrm{~cm}$, totalizando neste caso, seis amostras distintas;

3) Para a amostragem, cravou-se um tubo de PVC com $100 \mathrm{~mm}$ de diâmetro e $60 \mathrm{~cm}$ de altura, e coletou-se manualmente todo material contido no interior do tubo;

4) Todo material coletado foi seco ao ar, durante um período de aproximadamente $48 \mathrm{~h}$;

5) Após a secagem, o material coletado foi pesado e passado por peneiras padronizadas segundo as normas da ABNT, com diâmetros de abertura de $19,1,12,7,9,25,6,35,4,76$ e 2,38 mm. No processo de separação também foi utilizada a vibração mecânica. Determinou-se a massa do material retido em cada uma das peneiras e no recipiente de coleta dos finos, a fim realizar uma caracterização granulométrica do material constituinte do meio poroso;

6) O material retido no recipiente de coleta dos finos foi passado, ainda, por mais duas peneiras, também padronizadas segundo as normas da ABNT, com diâmetros de abertura de 1,0 e 0,212 $\mathrm{mm}$. O material retido na peneira de $0,212 \mathrm{~mm}$ (material grosseiro) e o retido no recipiente de coleta dessa peneira (material fino) foram separados em diferentes recipientes;

7) O restante do material, ou seja, com diâmetros $\geq 1,0 \mathrm{~mm}$, foi lavado com água destilada, utilizandose um volume máximo de $1,5 \mathrm{~L}$ por amostra de meio poroso, de forma a proporcionar a maior remoção possível do material aderido às britas. Para isso, utilizou-se uma bacia e uma peneira com malha de 1,3 $\mathrm{mm}$, para facilitar o procedimento;

8) O líquido resultante da lavagem de cada uma das amostras foi transferido para béqueres de 2,0 L, passando antes pela peneira de $1,0 \mathrm{~mm}$ de diâmetros de abertura, e levados para redução do teor de água em placas aquecedoras, sob temperatura de $60{ }^{\circ} \mathrm{C}$;

9) $\mathrm{O}$ material contido nos béqueres permaneceu nas chapas até que fosse obtida uma espécie de "lama". A partir desse ponto, essa "lama" foi transferida para um recipiente de alumínio e levada para secagem, em estufa, a $65{ }^{\circ} \mathrm{C}$, por 24 h. Após serem secadas, todas as amostras foram destorroadas com auxílio de um almofariz, passadas novamente na peneira de malha 0,212 $\mathrm{mm}$, e adicionada ao material grosseiro e fino separados inicialmente (item 7);

10) Ao final, após observar que ainda havia material aderido às pequenas britas da fração grosseira, optou-se por analisar separadamente grosseiros e finos;

11) Foram realizadas as seguintes análises nos materiais grosseiros e finos: conteúdo de água $\left(\mathrm{U}_{\mathrm{bs}}\right)$, pelo método gravimétrico, com secagem a $105-110{ }^{\circ} \mathrm{C}$ em estufa; sólidos voláteis totais (SVT) e sólidos fixos totais (SFT), após a calcinação do material obtido na secagem anterior, em mufla, sob temperatura de $550{ }^{\circ} \mathrm{C}$, por $2,0 \mathrm{~h}$, conforme constam no Standard Methods for the Examination of Water and Wasterwater (APHA et al., 2012);

12) Fez-se também uma análise de Raio- $X$ no material recolhido após a calcinação em mufla, sob a temperatura de $550{ }^{\circ} \mathrm{C}$ (item 12), a fim de identificar qualitativamente os minerais presentes no material retirado do leito dos SACs-EHSS e identificar se a brita também contribuiu para composição desse material colmatante. O material foi analisado em um Difratômetro com radiação de cobalto (Co), com comprimento de onda de $0,17902 \mathrm{~nm}$, faixa de varredura de $(2 \theta) 4$ a $70^{\circ} \mathrm{e}$ velocidade de varredura com um passo de $0,01^{\circ}$ a cada segundo. 
Todo procedimento descrito anteriormente, para amostragem e análise da brita, também foi realizado no material poroso coletado em um SAC-EHSS, de mesmas dimensões e preenchido com mesmo substrato que as demais unidades experimentais, mas que não sofreu nenhum tipo de tratamento, denominado SAC-EHSS 0. Dessa forma, foi possível identificar se houve ou não mudanças qualitativas e quantitativas nas características dos sólidos retidos no meio poroso dos SACs-EHSS, ao longo do tempo de condução do experimento.

Os dados de conteúdo de sólidos apresentados na razão massa por volume de poros $\left(\mathrm{kg} \mathrm{m}^{-3}\right)$, para serem apresentados em relação à matéria seca, foram submetidos às seguintes transformações:

$$
\begin{aligned}
& C_{S T}=\frac{M_{u}}{1+U_{b s}} \cdot \frac{1}{V \cdot \varepsilon} \\
& C_{S V T}=\frac{M_{u}}{1+U_{b s}} \cdot \frac{S V T}{100} \cdot \frac{1}{V \cdot \varepsilon} \\
& C_{S F T}=\frac{M_{u}}{1+U_{b s}} \cdot \frac{S F T}{100} \cdot \frac{1}{V \cdot \varepsilon}
\end{aligned}
$$

em que,

$\mathrm{C}_{\mathrm{ST}}=$ massa de sólidos totais por volume de meio poroso do SAC-EHSS $\left(\mathrm{kg} \mathrm{m}^{-3}\right)$;

$\mathrm{C}_{\mathrm{SVT}}=$ massa de sólidos voláteis totais por volume de meio poroso do SAC-EHSS $\left(\mathrm{kg} \mathrm{m}^{-3}\right)$;

$\mathrm{C}_{\mathrm{SFT}}=$ massa de sólidos fixos totais por volume de meio poroso do SAC-EHSS $\left(\mathrm{kg} \mathrm{m}^{-3}\right)$;

$\mathrm{M}_{\mathrm{u}}=$ massa seca ao ar do total de sólidos retirados do meio poroso $(\mathrm{kg})$;

$\mathrm{U}_{\mathrm{bs}}=$ conteúdo de água, em base seca, da massa dos sólidos retirados do meio poroso $\left(\mathrm{kg} \mathrm{kg}^{-1}\right)$;

SVT $=$ sólidos voláteis totais na amostra de sólidos $\operatorname{secos}\left(\right.$ dag $\left.\mathrm{kg}^{-1}\right)$;

SFT $=$ sólidos fixos totais na amostra de sólidos $\operatorname{secos}\left(\right.$ dag $\left.\mathrm{kg}^{-1}\right)$;

$\mathrm{V}=$ volume coletado do meio poroso $\left(\mathrm{m}^{3}\right)$; e

$\varepsilon=$ porosidade do meio $\left(\mathrm{m}^{3} \mathrm{~m}^{-3}\right)$.

Para obter quanto da $\mathrm{C}_{\mathrm{ST}}$ era composta por $\mathrm{C}_{\mathrm{SVT}}$ ou por $\mathrm{C}_{\mathrm{SFT}}$, em percentagem, fez-se as seguintes relações:

$$
\begin{aligned}
& \frac{C_{S V T}}{C_{S T}} .100 \\
& \frac{C_{S F T}}{C_{S T}} .100
\end{aligned}
$$

Para a análise granulométrica da brita, fezse uma análise de variância, considerando 7 tratamentos (SACs-EHSS 1i, 2i, 3i, 4ni, 5ni, 6ni e a testemunha, o SAC-EHSS 0), com 3 repetições (pontos de coleta do leito), no delineamento inteiramente casualizado (DIC). As médias foram comparadas utilizando-se o teste de Dunnett, para comparar os tratamentos com a testemunha, em nível de 5\% de probabilidade. Foi utilizado o programa computacional $\mathrm{R}$ ( $R$ CORE TEAM, 2014).

Os dados de sólidos intersticiais foram estudados por meio da estatística descritiva, com base nos fenômenos físicos, químicos e biológicos observados, além das informações fornecidas pelos dados coletados e analisados ao longo do período experimental.

\section{RESULTADOS E DISCUSSÃO}

No Quadro 3 estão apresentados os resultados das análises de variância, para cada abertura de peneira. $\mathrm{O}$ resultado do teste de médias, para o teste de Dunnett, está apresentado no Quadro 4.

Com base nos resultados apresentados nos Quadros 3 e 4, verifica-se que apenas a granulometria do substrato de preenchimento dos SACs-EHSS 4ni e 6ni diferiram em relação à da testemunha, para a peneira de $1,0 \mathrm{~mm}$ de abertura e para o recipiente de finos, sendo os valores médios de massa retida menores que o obtido no material poroso coletado no SAC-EHSS 0. Como essas diferenças foram ao acaso, não é possível identificar uma tendência mais específica nos resultados, isto é, não se pode inferir se as condições ambientais internas e externas ao sistema provocaram ou não a desintegração do substrato de preenchimento dos SACs-EHSS, a ponto de alterar sua granulometria, dentro de um período de aproximadamente 2,3 anos de operação dos sistemas. Essas diferenças podem estar relacionadas à pouca homogeneização

\section{REVENG}


Quadro 3. Resumo da análise de variância relativa à granulometria, para os SACs-EHSS 1i, 2i, 3i, 4ni, 5ni e 6 ni.

\begin{tabular}{|c|c|c|c|c|c|c|c|c|c|c|}
\hline \multirow[b]{2}{*}{$\begin{array}{l}\text { Fonte de } \\
\text { variação }\end{array}$} & \multirow[b]{2}{*}{ GL } & \multicolumn{9}{|c|}{ Quadrados médios para cada abertura de peneira } \\
\hline & & 19,10 & 12,70 & 9,25 & 6,35 & 4,76 & 2,38 & 1,00 & 0,212 & $\begin{array}{c}\text { Recipiente } \\
\text { de finos }\end{array}$ \\
\hline Tratamento & 6 & $0,378^{\text {ns }}$ & $1,936^{\text {ns }}$ & $15,788^{\text {ns }}$ & $2,379^{\text {ns }}$ & $1,180^{*}$ & $3,201^{\text {ns }}$ & $1,493 *$ & $0,305^{*}$ & $0,933 *$ \\
\hline Resíduo & 14 & 0,590 & 1,041 & 8,039 & 2,211 & 0,366 & 3,877 & 0,487 & 0,093 & 0,144 \\
\hline CV $(\%)$ & & 38,5 & 24,3 & 9,7 & 8,7 & 3,9 & 8,6 & 13,7 & 14,6 & 17,9 \\
\hline
\end{tabular}

*: significativo em nível de 5\%, pelo teste F. ns: não significativo em nível de 5\%, pelo teste F. GL - grau de liberdade; CV coeficiente de variação. Abertura das peneiras dada em mm.

Quadro 4. Média das percentagens de massa retida em relação a cada abertura de peneira, para os SACEHSS 1i, 2i, 3i, 4ni, 5ni e 6ni.

\begin{tabular}{|c|c|c|c|c|c|c|c|c|c|}
\hline \multirow[b]{2}{*}{ Tratamento } & \multicolumn{9}{|c|}{ Abertura das Peneiras (mm) } \\
\hline & 19,10 & 12,70 & 9,25 & 6,35 & 4,76 & 2,38 & 1,00 & 0,212 & $\begin{array}{c}\text { Recipiente } \\
\text { de finos }\end{array}$ \\
\hline SAC-EHSS 0 & 1,62 & 5,08 & 25,62 & 18,26 & 15,91 & 23,30 & 5,58 & 2,11 & 2,50 \\
\hline SAC-EHSS 1I & 1,92 & 3,69 & 31,92 & 16,06 & 15,31 & 21,94 & 5,03 & 2,08 & 2,03 \\
\hline SAC-EHSS 2I & 2,18 & 4,10 & 28,76 & 18,00 & 14,89 & 22,51 & 5,38 & 2,47 & 1,70 \\
\hline SAC-EHSS 3I & 2,03 & 5,29 & 30,21 & 16,52 & 15,14 & 21,96 & 4,94 & 1,58 & 2,32 \\
\hline SAC-EHSS 4NI & 1,71 & 4,59 & 29,48 & 17,29 & 15,91 & 22,57 & $3,61^{*}$ & 1,76 & 3,08 \\
\hline SAC-EHSS 5NI & 2,67 & 3,46 & 31,26 & 16,02 & 14,86 & 22,70 & 5,27 & 2,16 & 1,60 \\
\hline SAC-EHSS 6 NI & 1,81 & 3,23 & 26,78 & 17,00 & 16,54 & 24,94 & 5,74 & 2,40 & $1,56^{*}$ \\
\hline
\end{tabular}

SAC-EHSS 0: testemunha. As médias com * na mesma coluna diferem da testemunha em nível de $5 \%$ de significância, pelo teste de Dunnett.

da brita, quando do preenchimento dos SACsEHSS, já que o material é uma mistura de brita \# 0 com brita \# 1 .

Apesar disso, Caselles-Osorio e Garcia (2006) ressaltaram que a formação de ácidos ligada às vias bioquímicas de remoção de matéria orgânica pode promover a dissolução e desintegração do meio granular em SACs. Esta desintegração também pode ocorrer por agentes físicos, como uso de maquinários (KNOWLES et al., 2011). O tipo de material que compõe o leito dos SACs também pode, naturalmente, ser mais susceptível ao desgaste. Por exemplo, a brita utilizada para preencher os SACs-EHSS é composta por gnaisse, uma rocha metamórfica que, segundo Fontes (2012), apresenta, em geral, estruturas típicas como xistosidade, foliação gnáissica e estratificação e que, usualmente, provocam menor resistência ao intemperismo ou sua desagregação. O gnaisse é composto por mais de $20 \%$ de feldspato potássico (microclínio e/ou ortoclásio), além de plagioclásio (albita, oligoclásio, andesina), quartzo e biotita (MACHADO, 2003). Seus constituintes menores são clorita, anfibólio, granada, estaurolita, sillimanita, cianita, carbonato, cordierita, espinélio verde, opacos, titanita e epidoto (MACHADO, 2003). Muitos desses minerais foram detectados nas amostras, por meio da análise de Raio-X e, em todos os casos, há presença de quartzo e feldspatos. A presença de biotita foi menos pronunciada no material mais grosseiro.

A presença de minerais, constituintes do gnaisse, nas amostras analisadas indica que grande parte dos sólidos fixos é composta pelo 
pó oriundo da brita utilizada como substrato. A maior parte deste pó foi, provavelmente, produzida pelo atrito das pedras durante a manipulação do material, quando da montagem do experimento. Pedescoll et al. (2009) também demonstraram, por meio de análises de difração de Raio-X, que a composição mineral dos sólidos acumulados no SAC-EHSS estudados foi semelhante à do material que compunha o leito (principalmente quartzo e calcita), indicando que a fração inorgânica do material colmatante pode ter origem no pó presente no substrato. Segundo Pedescoll et al. (2011), a utilização de substratos de má qualidade para preenchimento de SACs-EHSS pode acelerar a sua colmatação. Os autores avaliaram o substrato utilizado em seis SACs-EHSS distintos, quanto à sua composição e as propriedades de resistência mecânica, e os resultados mostraram que tanto a mineralogia quanto a textura estão relacionadas à resistência mecânica.

Durante a coleta das amostras de brita foi observado que, ao retirar o tubo do interior do leito, o buraco formado permanecia praticamente intacto, principalmente na camada mais superficial, o que caracteriza a presença de material estruturante no meio poroso dos SACs-EHSS estudados. Esse fenômeno não foi notado no sistema com brita limpa, cujo leito "desmoronava" quando era retirado o tubo. A matéria orgânica tem a capacidade de atuar como agente ligante ou desagregante, dependendo de sua composição química e da presença de outros materiais cimentantes (SILVA et al., 2010). Silva e Mendonça (2007) explicaram que a matéria orgânica particulada, hifas de fungos e raízes de plantas podem formar um emaranhado de microagregados. A morte das raízes e o crescimento das hifas, dentro e entre macroagregados, produzem agentes ligantes bioquímicos capazes de estabilizálos. Embora a agregação de sólidos dispersos seja um processo dinâmico, já que desagregação pode ocorrer por ação microbiana sobre o material orgânico cimentante das partículas primárias, os autores explicaram que o aporte continuado de material orgânico, tal como ocorre em SACsEHSS, leva a um balanço positivo, garantindo a agregação do meio poroso.

Os resultados das análises dos sólidos finos e grosseiros retidos nos interstícios do meio poroso e referentes a todo perfil do meio poroso dos leitos (camada de 0 a $55 \mathrm{~cm}$ ) estão apresentados na forma de histogramas na Figura 1.

Em relação aos sistemas em que não houve a inversão no sentido do escoamento da água residuária, $\mathrm{C}_{\mathrm{ST}}$ na região de saída foi maior que na região central e, em alguns casos, maior que na zona de entrada, tanto para os materiais finos quanto para os grosseiros. Excepcionalmente, no SAC-EHSS 4, esse comportamento não foi verificado em relação aos grosseiros retidos nos interstícios do meio poroso.

Esperava-se, no entanto, que $\mathrm{C}_{\mathrm{ST}}$ fosse decrescente da entrada para a saída dos SACsEHSS em que não houve a inversão no sentido do escoamento, já que a região inicial funcionou como um filtro. Uma possível explicação para essa distribuição de $\mathrm{C}_{\mathrm{ST}}$ ao longo dos SACs-EHSS, nos quais o sentido do escoamento não foi invertido seria de que na região de saída da água residuária dos sistemas há afunilamento da passagem de água, formando uma zona de estagnação, o que favorece a retenção de sólidos nos poros. Além disso, durante a fase de montagem, os sistemas, já preenchidos com o substrato, foram cheios e esvaziados repetidas vezes com água para que se pudesse efetuar seu nivelamento a $50 \mathrm{~cm}$ de altura e para que alguns vazamentos pudessem ser reparados. Isso pode ter provocado o carreamento de partículas sólidas para a região de saída dos SACs-EHSS.

Caselles-Osorio et al. (2007) também esperavam maior acúmulo de sólidos totais $\left(\mathrm{kg}_{\text {matéria }}\right.$ $\mathrm{m}_{\text {seca }} \mathrm{m}^{-2}$ ) próximos à entrada dos SACs, no entanto, observaram distribuição bastante heterogênea na maioria dos SACs-EHSS estudados. O mesmo foi observado no trabalho de Pedescoll et al. (2013), que explicaram que a água tende a fluir ao longo de caminhos com menor resistência e, assim, as regiões onde ocorre escoamento preferencial há maior acúmulo de sólidos totais, ao longo do tempo. Pedescoll et al. (2009) encontraram maior quantidade de sólidos totais $\left(\mathrm{kg} \mathrm{m}^{-2}\right)$ na região de saída em um dos SACs-EHSS estudados. Paoli e von Sperling (2013), contrariamente ao que foi verificado neste trabalho, observaram conteúdo de sólidos totais $\left(\mathrm{g} \mathrm{L}^{-1}\right)$ decrescente da entrada para a saída, em SAC-EHSS plantado.

Quanto à distribuição de $\mathrm{C}_{\mathrm{SVT}}$, observa-se 


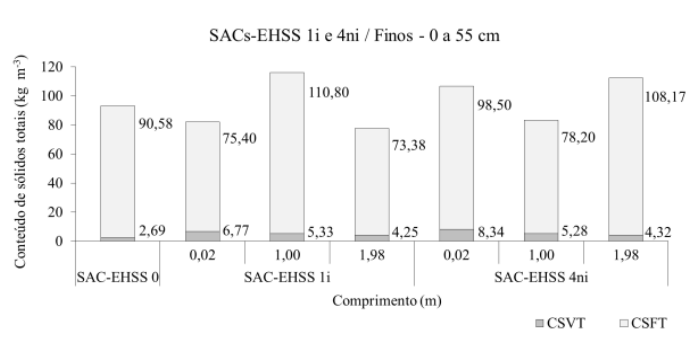

(a)

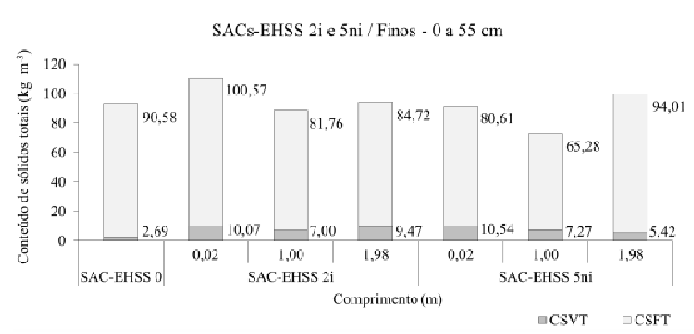

(c)

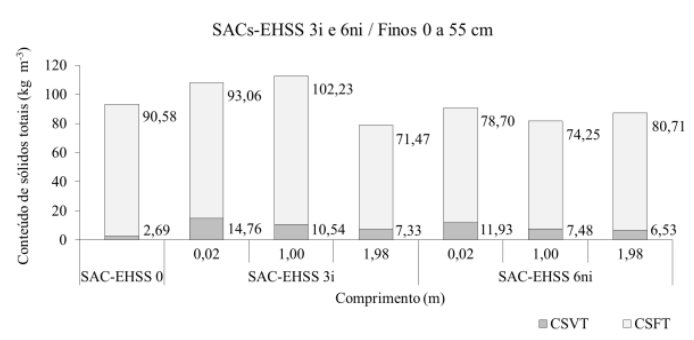

(e)

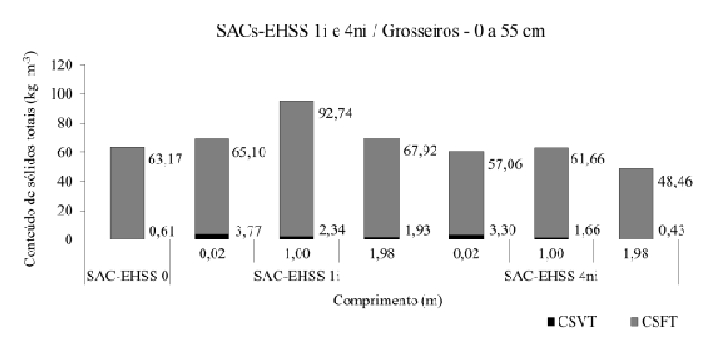

(b)

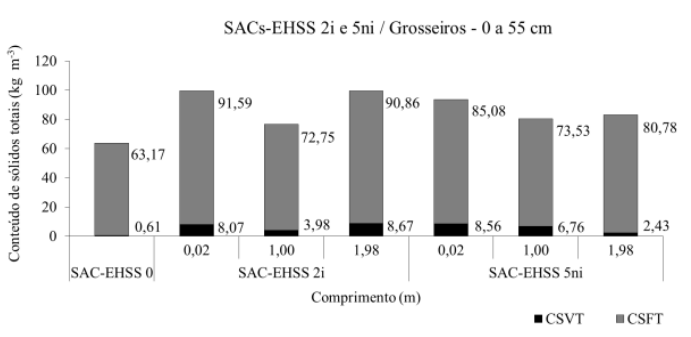

(d)

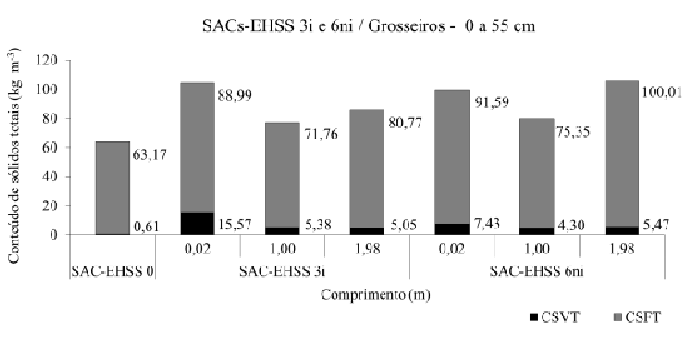

$(f)$

Figura 1. Conteúdo (massa seca por volume de poros) de sólidos totais $\left(\mathrm{C}_{\mathrm{ST}}\right)$, sólidos totais fixos $\left(\mathrm{C}_{\mathrm{STF}}\right)$ e sólidos totais voláteis $\left(\mathrm{C}_{\mathrm{STV}}\right)$, em $\mathrm{kg} \mathrm{m}^{-3}$, média de todo o perfil (camada de 0 a $55 \mathrm{~cm}$ ), nos SAC-EHSS 0, testemunha e distribuídos ao longo do comprimento dos SACs-EHSS 1i e 4ni, sem cultivo, em relação aos sólidos finos $(a)$ e grosseiros (b); SACs-EHSS $2 \mathrm{i}$ e 5ni, cultivados com capim tifton 85, em relação aos sólidos finos (c) e grosseiros $(d)$; e SACs-EHSS 3 i e 6ni, cultivados com alternantera, em relação aos sólidos finos $(e)$ e grosseiros $(f)$. ${ }^{*}$ índice $i$ referese a sistemas com inversão no sentido do escoamento e o índice $n i$ a sistemas sem inversão no sentido do escoamento.

tendência de sua redução, bem como da relação $\mathrm{C}_{\mathrm{SVT}} / \mathrm{C}_{\mathrm{ST}}$, da entrada para a saída dos SACs-EHSS, ao se considerar a média do material coletado em todo o perfil poroso, ou seja, na camada de 0 a $55 \mathrm{~cm}$. Assim, apesar da saída dos sistemas não invertidos apresentarem maior $\mathrm{C}_{\mathrm{ST}}$, possuem menores $\mathrm{C}_{\mathrm{SVT}}$ e $\mathrm{C}_{\mathrm{SVT}} / \mathrm{C}_{\mathrm{ST}}$. Paoli e von Sperling (2013) também observaram valores decrescentes de conteúdo de sólidos voláteis $\left(\mathrm{g} \mathrm{L}^{-1}\right)$ do início para o final de SACs-EHSS plantados e não plantados, no entanto, a relação sólidos voláteis/sólidos totais não seguiu a mesma tendência. Tanner \& Sukias (1995) reportaram uma variação do acúmulo de sólidos voláteis de $9,5 \mathrm{~kg} \mathrm{~m}^{-2}$, na entrada, para 2 e $5 \mathrm{~kg} \mathrm{~m}^{-2}$, na saída, no SAC-EHSS plantado.

Em média, nos SACs-EHSS que não tiveram inversão no sentido do escoamento, a relação $\mathrm{C}_{\mathrm{SVT}} / \mathrm{C}_{\mathrm{ST}}$, para entrada, meio e saída foi de, respectivamente, 10,$8 ; 8,5$ e $5,6 \%$ nos materiais finos, e de 7,4; 5,5 e 3,0\% nos mais grosseiros. Considerando a relação $\mathrm{C}_{\mathrm{SVT}} j \mathrm{C}_{\mathrm{ST}}$ para cada amostra do material poroso do leito dos SACs-EHSS que não tiveram inversão no sentido do escoamento, os valores variaram de 0,9 a 13,2\%, entre finos e grosseiros. Embora uma comparação com dados apresentados na literatura fique difícil, tendo em 
vista que a metodologia de extração dos sólidos e as unidades utilizadas para as análises de sólidos intersticiais não são padronizadas, o que pode provocar variações nos resultados, Paoli e von Sperling (2013) obtiveram de 22 e $20 \%$ de SVT nos sólidos coletados nos poros, respectivamente, de SACs-EHSS plantados e não plantados; Caselles-Osorio et al. (2007) de 10 a $20 \%$ em SACs-EHSS plantados; e Tanner \& Sukias (1995), de 15 e $8 \%$, respectivamente, em SACs-EHSS plantados e não plantados. Pedescoll et al. (2009) relataram que as porcentagens de sólidos voláteis no material colmatante variaram de 15 a $33 \%$ e que a quantidade de material inorgânico encontrado nos sólidos retidos no leito foi superior a $75 \%$, nos seis SAC-EHSS estudados, todos cultivados.

Em relação aos sistemas com inversão no sentido do escoamento da água residuária, de maneira geral, a técnica levou ao maior acúmulo de sólidos totais $\left(\mathrm{C}_{\mathrm{ST}}\right)$ e matéria orgânica $\left(\mathrm{C}_{\mathrm{SVT}}\right)$ no meio poroso, considerando-se uma média para todo o perfil. $\mathrm{O}$ valor de $\mathrm{C}_{\mathrm{SVT}}$ foi decrescente da região 1 para a 3, nos SACs-EHSS 1i e 3i, tal qual nos SACs-EHSS 4ni e 6ni, tanto para o material fino quanto para o grosseiro. Notou-se, também, maior $\mathrm{C}_{\mathrm{SVT}}$ na região 3 dos sistemas invertidos, em relação aos sistemas não invertidos, no que diz respeito ao material fino e grosseiro. A região 3 passou a receber a ARS após a inversão no sentido do escoamento e, assim, maior aporte de matéria orgânica, promovendo-se seu maior acúmulo no meio poroso dessa região.

No meio poroso do SAC-EHSS 1i, sem cultivo, e do SAC-EHSS $2 \mathrm{i}$, cultivado com capim tifton 85 , que passaram mais tempo sob regime de inversão no sentido do escoamento (aproximadamente 14 e 10 meses, respectivamente), ocorreu redução de $\mathrm{C}_{\mathrm{SVT}}$ na região 1 , quando comparados ao $\mathrm{C}_{\mathrm{SVT}}$ na região 1 dos SAC-EHSS 4ni, sem cultivo, e do SAC-EHSS 5ni, cultivado com capim tifton, respectivamente. Todavia, essas reduções não comprovaram a hipótese de que, com a inversão, haveria descolmatação do meio poroso.

Em Mai/2013, após apresentarem um alcance do ES de 2,0 m, os SACs-EHSS 1i e 4ni foram desativados, permanecendo em repouso até o momento da coleta das amostras do material poroso do leito. Acredita-se que a maior redução de $\mathrm{C}_{\mathrm{SVT}}$, que alcançou $18,9 \%$ na região 1 do SACEHSS 1i, sem cultivo, esteja associada a esse período de repouso pelo qual o sistema passou. $\mathrm{Na}$ comparação entre os valores de $\mathrm{C}_{\mathrm{SVT}}$ obtidos no meio poroso do SAC-EHSS $2 \mathrm{i}$ e 5ni, cultivados com capim tifton 85 , essa redução foi menor $(4,5 \%$ para os finos e de 5,7\% para os grosseiros), o que decorre do pouco tempo de manutenção da nova condição de operação dos sistemas (sentido do escoamento invertido). Nos SACs-EHSS $3 \mathrm{i}$ e 6ni, cultivados com alternantera, não houve redução de $\mathrm{C}_{\mathrm{SVT}}$ no meio poroso da região 1 . Acredita-se que, se a inversão tivesse ocorrido mais prematuramente, todos os sistemas invertidos apresentariam maiores reduções nos valores de $\mathrm{C}_{\mathrm{SVT}}$, na região 1 .

Os resultados das análises dos sólidos finos e grosseiros, retidos nos interstícios do meio poroso e analisados por camada (superficial, de 0 a $20 \mathrm{~cm}$ e subsuperficial, de 20 a $55 \mathrm{~cm}$ ) do meio poroso estão apresentados, na forma de histogramas, na Figura 2.

Quando se analisa separadamente o meio poroso dos SACs-EHSS plantados e não invertidos por camadas, ou seja, de 0 a $20 \mathrm{~cm}$ e de 20 a 55 $\mathrm{cm}$, observa-se comportamento distinto no que se refere aos resultados obtidos quando da análise referente a todo o perfil.

$\mathrm{Na}$ camada de 0 a $20 \mathrm{~cm}$, verificou-se tendência de redução em $\mathrm{C}_{\mathrm{SVT}}$ e na relação $\mathrm{C}_{\mathrm{SVT}} / \mathrm{C}_{\mathrm{ST}}$, do início para o fim dos SACs-EHSS. No entanto, a região de saída não necessariamente apresentou o menor valor de $\mathrm{C}_{\mathrm{ST}} \mathrm{e}$, em alguns casos, apresentou valores intermediários.

Com relação à camada de $20 \mathrm{a} 55 \mathrm{~cm}$, ocorreu redução em $\mathrm{C}_{\mathrm{SVT}}$ e na relação $\mathrm{C}_{\mathrm{SVT}} / \mathrm{C}_{\mathrm{ST}}$ da região de entrada para região de saída dos SACs-EHSS, embora o mesmo comportamento não tenha sido observado no meio poroso do SAC-EHSS 5ni, cultivado com capim tifton 85 , já que, nesse sistema, a maior $\mathrm{C}_{\mathrm{SVT}}$ esteve presente na região central.

A explicação para essa redução é a de que a matéria orgânica que chega à região de saída já passou por vários processos de decomposição ao longo do SAC-EHSS, sendo, portanto, um material mais recalcitrante e em menor quantidade. Por isso há decréscimo na $\mathrm{C}_{\mathrm{SvT}}$ à medida que se caminha da entrada para a saída, na camada de $20-55 \mathrm{~cm}$ dos 


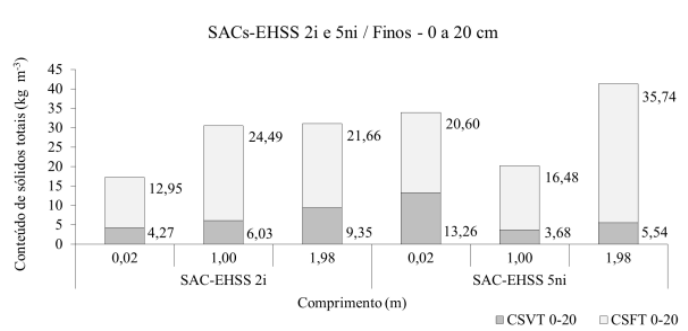

(a)

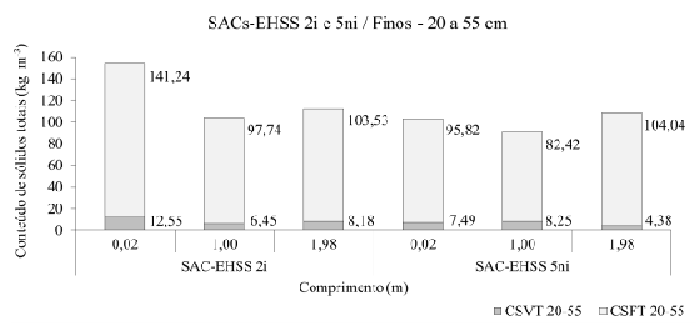

(c)

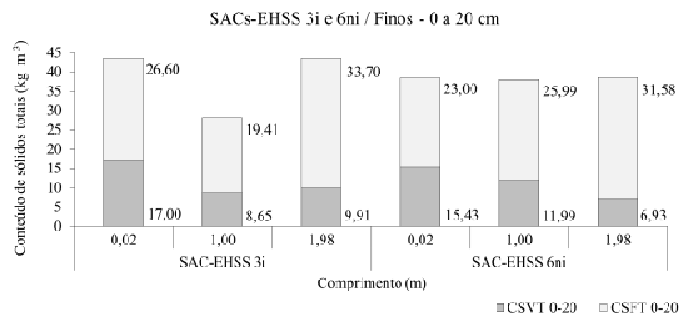

(e)

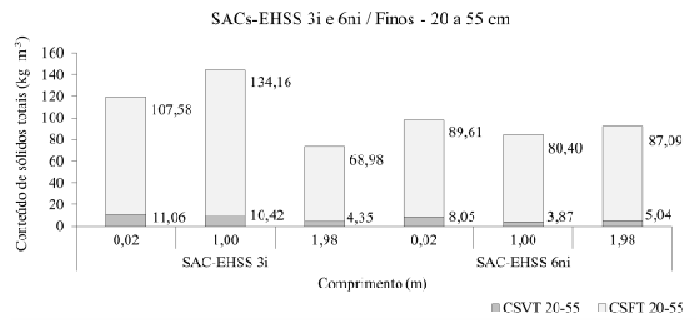

$(g)$

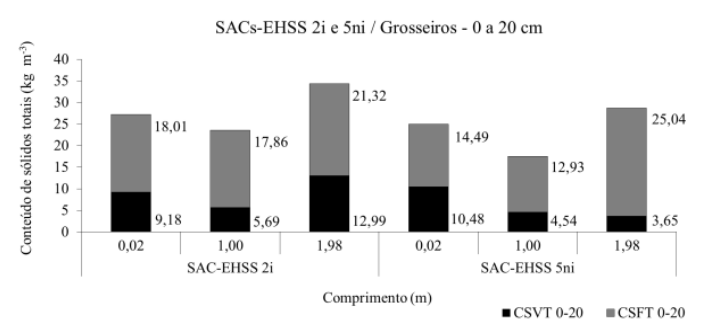

(b)

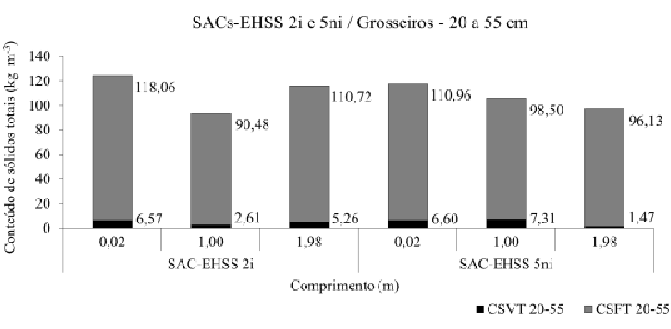

(d)

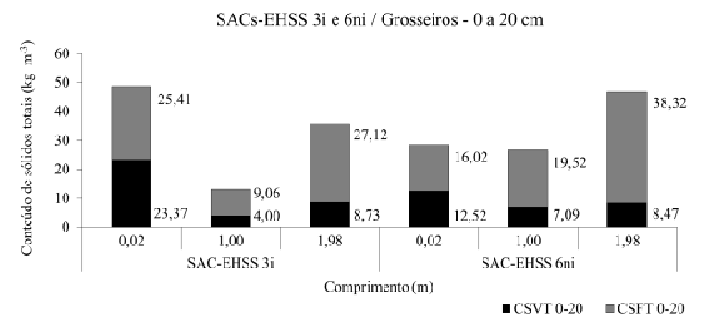

$(f)$

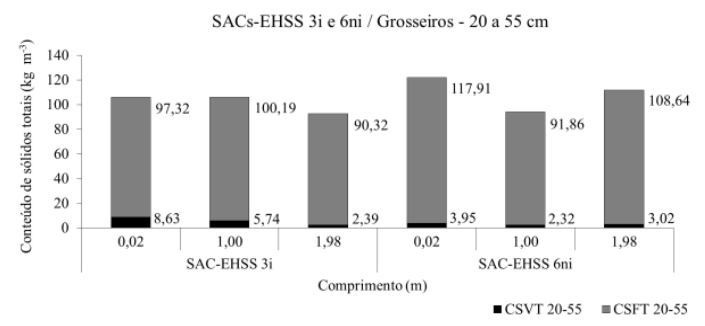

(h)

Figura 2. Conteúdo (massa seca por volume de poros) de sólidos totais $\left(\mathrm{C}_{\mathrm{ST}}\right)$, sólidos totais fixos $\left(\mathrm{C}_{\mathrm{STF}}\right)$ e de sólidos totais voláteis $\left(\mathrm{C}_{\mathrm{STV}}\right)$, em $\mathrm{kg} \mathrm{m}^{-3}$, distribuídos ao longo do comprimento dos SACsEHSS $2 \mathrm{i}$ e 5ni, cultivados com capim tifton 85 , na camada de $0-20 \mathrm{~cm}$, em relação aos sólidos finos $(a)$ e grosseiros $(b)$, e na camada de $20-55 \mathrm{~cm}$, em relação aos sólidos finos $(c)$ e grosseiros (d); nos SACs-EHSS $3 i$ e 6ni, cultivados com alternantera, na camada de 0-20 cm, em relação aos sólidos finos $(e)$ e grosseiros $(f)$, e na camada de $20-55 \mathrm{~cm}$, em relação aos sólidos finos $(g)$ e grosseiros $(h)$. ${ }^{*} \mathrm{O}$ índice $i$ refere-se aos sistemas com inversão no sentido do escoamento e o índice $n i$ a sistemas sem inversão no sentido do escoamento.

SAC-EHSS não invertidos.

Os resultados das análises de sólidos, referentes à camada de 0 a $20 \mathrm{~cm}$ dos sistemas plantados, destacam-se dos demais pelos valores de $\mathrm{C}_{\mathrm{SVT}}$ e relação $\mathrm{C}_{\mathrm{SVT}} / \mathrm{C}_{\mathrm{ST}}$ que foram, na maioria das vezes, maiores que os obtidos na camada de 20 a $55 \mathrm{~cm}$. Isso decorre do fato de que na camada superficial do meio poroso é que se encontram a maioria das 
raízes e depósitos de detritos vegetais, além da contribuição do biofilme e dos sólidos da água residuária (TANNER; SUKIAS, 1995; KNOWLES et al., 2011; NIVALA et al., 2012), o que aumenta a proporção de SVT no material acumulado nos poros. No trabalho de Nguyen (2000), em SACEHSS cultivado, a maior parte da matéria orgânica acumulada na camada superficial de $100 \mathrm{~mm}$ foi oriunda da morte de raízes e rizomas, exsudação de compostos orgânicos a partir das raízes e a grande quantidade de biomassa e atividade microbiana. Os autores estimaram que, aproximadamente, $27 \%$ do bloqueio dos poros nessa camada deviase à presença de raízes e rizomas das plantas, $73 \%$ foi causado por depósitos orgânicos proveniente de água residuária aplicada e de detritos da parte aérea das plantas, e apenas $4 \%$ do bloqueio dos poros deveu-se a depósitos inorgânicos. Segundo o mesmo autor, mais de $90 \%$ da matéria orgânica acumulada no leito era composta por frações recalcitrantes. No trabalho de Nivala et al. (2007), a colmatação se deu, principalmente, devido ao acúmulo de precipitados de ferro.

Por ser a colmatação um fenômeno predominantemente superficial (XIE et al., 2010), a opção de aplicar o afluente subsuperficialmente evitaria o acúmulo dos sólidos da água residuária sobre o leito. Segundo Knowles et al. (2011), a aplicação de água residuária de forma subsuperficial promove um aproveitamento mais eficiente de todo o perfil do meio poroso do SAC-EHSS,

Em relação aos efeitos da inversão no sentido do escoamento na quantidade de material orgânico acumulado nos poros dos sistemas, verificou-se que, na região 3 do SAC-EHSS 2i, cultivado com capim tifton 85 , tanto nas camadas de $0-20$ como de 20-55 cm, proporcionou aumento de $\mathrm{C}_{\mathrm{SVT}}$ entre o material fino e grosseiro. Entretanto, na região 1 houve redução em $\mathrm{C}_{\mathrm{SVT}}$, na camada de $0-20 \mathrm{~cm}$, para os finos e grosseiros. Isso demonstrou efeitos positivos da inversão no sentido de escoamento da água residuária, que pode estar associado à falta de ocorrência de $E S$ nessa região do leito, deixando a camada superficial mais passível de aeração e, com isso, facilitando a degradação do material acumulado, já que subsuperficialmente a redução da $\mathrm{C}_{\mathrm{SVT}}$ não ocorreu ou não foi expressiva.

Segundo Molle et al. (2006), a chave para assegurar a longevidade operacional dos SACs é otimizar o arejamento dos poros, para garantir condições aeróbicas, controlar o crescimento da biomassa aderida e a taxa de mineralização dos sólidos suspensos retidos.

$\mathrm{Na}$ camada de $0-20 \mathrm{~cm}$ do SAC-EHSS 3i, cultivado com alternantera, o $\mathrm{C}_{\mathrm{SVT}}$, no que se refere a sólidos finos e grosseiros, ocorreu de forma semelhante ao que foi verificado ao longo do comprimento do leito, considerando todo o perfil. No entanto, na camada de $20-55 \mathrm{~cm}$, o maior valor de $\mathrm{C}_{\mathrm{SVT}}$ ocorreu nas regiões 1 e 2 . Após a inversão no sentido do escoamento no SAC-EHSS 3i, a ARS permaneceu empoçada em toda extensão superficial sistema e, assim, os sólidos da água residuária foram depositados sobre a superfície, promovendo maiores mudanças nas condições hidrodinâmicas da camada superficial. De acordo com Pedescoll et al. (2013), a parte superior do meio poroso, principalmente na zona de entrada dos SACs-EHSS, forma o primeiro filtro para os sólidos da água residuária aplicada. E dessa forma, se colmata mais rápido.

Percebe-se que a inversão no sentido do escoamento da água residuária afetou mais a camada superficial do meio poroso, devido à falta de ocorrência de $E S$, deixando evidente a importância da época de se efetuar essa inversão, para que se possa alcançar em magnitude e amplitude os efeitos positivos desse procedimento operacional de descolmatação do meio poroso de SACs-EHSS.

\section{CONCLUSÕES}

Com base nos resultados obtidos, pode-se concluir que:

- Ao fim de 2,3 anos de operação, não foi verificado efeito perceptível das condições ambientais, internas e externas ao sistema, na desintegração do substrato de preenchimento e acúmulo desses finos no meio poroso dos SACs-EHSS avaliados;

- A matéria orgânica, mesmo quando presente em baixas proporções (baixa relação $\mathrm{C}_{\mathrm{SVT}}$ ' $\mathrm{C}_{\mathrm{ST}}$ ) é capaz de fomentar a estruturação de partículas presentes na camada superficial do meio poroso dos SACs-EHSS, tendo eles sido

\section{REVENG


submetidos ou não à inversão no sentido do escoamento da água residuária;

- Em média, a inversão no sentido do escoamento não proporcionou redução noacúmulo de matéria orgânica no meio poroso dos SACsEHSS cultivados, principalmente na camada de 0 a $20 \mathrm{~cm}$;

- O alto grau de colmatação que já se encontravam os SACs-EHSS foi, decisivamente, o fator que impediu ou tornou menos perceptíveis e prolongados os efeitos positivos da inversão no sentido do escoamento da água residuária;

- Cada espécie vegetal influenciou de modo distinto a distribuição de $\mathrm{C}_{\mathrm{ST}}, \mathrm{C}_{\mathrm{SFT}}$ e $\mathrm{C}_{\mathrm{SVT}}$ nos SACs-EHSS plantados, devido aos diferentes padrões de crescimento que elas apresentam.

\section{AGRADECIMENTOS}

Ao Departamento de Engenharia Agrícola (DEA) da Universidade Federal de Viçosa (UFV), à Fundação de Amparo à Pesquisa do Estado de Minas Gerais (FAPEMIG), ao Conselho Nacional de Desenvolvimento de Científico e Tecnológico (CNPq) e ao Grupo de Pesquisa em Qualidade Ambiental (GPQA).

\section{REFERÊNCIAS BIBLIOGRÁFICAS}

APHA (AMERICAN PUBLIC HEALTH ASSOCIATION); AWWA (AMERICAN WATER WORKS ASSOCIATION); WEF (WATER ENVIRONMENT FEDERATION). Standard methods for the examination of water and wastewater. $22^{\text {nd }}$ ed. Washington, D.C.: American Public Health Association, 2012. 1046p.

CASELLES-OSORIO, A.; GARCÍA, J. Performance of experimental horizontal subsurface flow constructed wetlands fed with dissolved or particulate organic matter. Water Research, v.40, n.19, p.3603-3611, 2006.

CASELLES-OSORIO, A.; PUIGAGUT, J.; SEGÚ, E.; VAELLO, N.; GRANÉS, F.; GARCÍA, D.; GARCÍA, J. Solids accumulation in six fullscale subsurface flow constructed wetlands. Water
Research, v.41, n.6, p.1388-1398, 2007.

CHAZARENC, F.; MERLIN, G. Influence of surface layer on hydrology and biology of gravel bed vertical flow constructed wetlands. Water Science and Technology, v.51, n.9, p.91-97, 2005.

FONTES, M.P.F. Intemperismo de rochas e minerais. In: KER, J.C.; CURI, N.; SCHAEFER, C.E.G.R. e VIDAL-TORRADO, P. (Eds.). Pedologia: Fundamentos. $1^{\text {a }}$. Viçosa, MG: Sociedade Brasileira de Ciência do Solo, 2012. cap. VI, 343p.

KADLEC, R.H.; WALLACE, S. Treatment wetlands. $2^{\text {nd }}$ ed. Florida: CRC press, 2008. 1016p.

KNOWLES, P.; DOTRO, G.; NIVALA, J.; GARCÍA, J. Clogging in subsurface-flow treatment wetlands: occurrence and contributing factors. Ecological Engineering, v.37, p.99-112, 2011.

MACHADO, F.B. Enciclopédia Multimídia de Minerais e Atlas e Rochas. Rio Claro, SP: Departamento de Petrologia e Metalogenia, UNESP, 2003. Disponível em: < http://www. rc.unesp.br/museudpm/banco/index.html Acesso em: 08 de outubro de 2014.

MOLLE, P.; LIÉNARD, A.; GRASMICK, A.; IWEMA, A. Effect of reeds and feeding operations on hydraulic behaviour of vertical flow constructed wetlands under hydraulic overloads. Water Research, v.40, n.3, p.606-612, 2006.

NGUYEN, L.M. Organic matter composition, microbial biomass and microbial activity in gravelbed constructed wetlands treating farm dairy wastewaters. Ecological Engineering, v.16, n.2, p.199-221, 2000.

NIVALA， J.; KNOWLES, P.; DOTRO, G.; GARCÍA, J.; WALLACE, S. Clogging in subsurface-flow treatment wetlands: measurement, modeling and management. Water Research, v.46, p.1625-1640, 2012.

PAOLI, A.C.; VON SPERLING, M. Evaluation 
of clogging in planted and unplanted horizontal subsurface flow constructed wetlands: solids accumulation and hydraulic conductivity reduction. Water Science and Technology, v.67, n.6, p.13451352, 2013.

PEDESCOLL, A.; PASSOS, F.; ALBA, E.; GARCIA, J.; PUIGAGUT, J. Mechanical resistance properties of gravel used in subsurface flow constructed wetlands: implications for clogging. Water Science and Technology, v.63, n.9, p.1801-1807, 2011.

PEDESCOLL, A.; SIDRACH-CARDONA, R.; SÁNCHEZ, J.C.; CARRETERO, J.; GARFI, M.; BÉCARES, E. Design configurations affecting flow pattern and solids accumulation in horizontal free water and subsurface flow constructed wetlands. Water Research, v.47, n.3, p.1448-1458, 2013.

PEDESCOLL, A.; UGGETTI, E.; LLORENS, E.; GRANÉS, F.; GARCÍA, D.; GARCÍA, J. Practical method based on saturated hydraulic conductivity used to assess clogging in subsurface flow constructed wetlands. Ecological Engineering, v.35, p.1216-1224, 2009.

\section{R CORE TEAM. R: A language and environment}

for statistical computing. Versão Vienna, Austria: R Fundation for Statistical Computing, 2014. Disponível em: < http://www.R-project.org/ > Acesso em: 05 de outubro de 2014.

SILVA, A.P.; TORMENA, C.A.; DIAS JUNIOR, M.S.; IMHOFF, S.; KLEIN, V.A. Indicadores da qualidade física do solo. In: VAN LIER, Q. J. (Ed.). Física do Solo. $1^{\text {a }}$. Viçosa, MG: Sociedade Brasileira de Ciência do Solo, 2010. cap. VII, 298p.

SILVA, I.R.; MENDONÇA, E.S. Matéria orgânica do solo. In: NOVAIS, R.F.; ALVEZ V., V.H.; BARROS, N.F.; FONTES, R.L.F.; CANTARUTTI, R.B. e NEVES, J.C.L. (Eds.). Fertilidade do solo. $1^{\text {a }}$. Viçosa, MG: Sociedade Brasileira de Ciência do Solo, 2007. cap. VI, 1017p..

TANNER, C.C.; SUKIAS, J.P. Accumulation of organic solids in gravel-bed constructed wetlands. Water Science and Technology, v.32, n.3, p.229239, 1995.

XIE, X.L.;HE, F.; XU, D.; WU, Z.B. Hydrodynamic aspects of particle clogging in the simulated vertical flow constructed wetland using river sands as substrate. Fresenius Environmental Bulletin, v.19, n.11, p.2567-2575, 2010. 